\title{
Functional links between motor and language systems
}

\author{
Friedemann Pulvermüller, ${ }^{1}$ Olaf Hauk, ${ }^{1}$ Vadim V. Nikulin ${ }^{2}$ and Risto J. Ilmoniemi ${ }^{3,4}$ \\ ${ }^{1}$ Cognition and Brain Sciences Unit, Medical Research Council, 15 Chaucer Road, Cambridge CB2 2EF, UK \\ ${ }^{2}$ Karolinska Institutet, Clinical Neurophysiology, Karolinska Hospital, Stockholm, Sweden \\ ${ }^{3}$ BioMag Laboratory, Engineering Centre, Helsinki University Central Hospital, Helsinki Brain Research Centre, Helsinki, Finland \\ ${ }^{4}$ Nexstim Ltd, Helsinki, Finland
}

Keywords: cell assembly, cognition, electromyography, mirror neurons, reaction time, transcranial magnetic stimulation

\begin{abstract}
Transcranial magnetic stimulation (TMS) was applied to motor areas in the left language-dominant hemisphere while right-handed human subjects made lexical decisions on words related to actions. Response times to words referring to leg actions (e.g. kick) were compared with those to words referring to movements involving the arms and hands (e.g. pick). TMS of hand and leg areas influenced the processing of arm and leg words differentially, as documented by a significant interaction of the factors Stimulation site and Word category. Arm area TMS led to faster arm than leg word responses and the reverse effect, faster lexical decisions on leg than arm words, was present when TMS was applied to leg areas. TMS-related differences between word categories were not seen in control conditions, when TMS was applied to hand and leg areas in the right hemisphere and during sham stimulation. Our results show that the left hemispheric cortical systems for language and action are linked to each other in a category-specific manner and that activation in motor and premotor areas can influence the processing of specific kinds of words semantically related to arm or leg actions. By demonstrating specific functional links between action and language systems during lexical processing, these results call into question modular theories of language and motor functions and provide evidence that the two systems interact in the processing of meaningful information about language and action.
\end{abstract}

\section{Introduction}

The traditional view has been that language primarily involves two core areas in the left inferior frontal and superior temporal cortex. These were considered modules for language production and comprehension, respectively (Lichtheim, 1885; Geschwind, 1970). With the advent of neuroimaging, however, the evidence became overwhelming that, apart from classic core language areas, multiple supplementary areas are also active during language processing, suggesting widely distributed cortical systems as the basis of language in the brain (Pulvermüller, 1996; Vandenberghe et al., 1996; Binder et al., 1997). To reconcile the classic modular theory with modern distributed accounts, one may claim that the core regions for language are crucial whereas activation of supplementary areas is a redundant by-product of language processing. However, an alternative possibility is that supplementary language areas are as critical as core areas but selective for specific categories of knowledge so that they primarily engage in processing specific types of words and concepts (Shallice, 1988; Pulvermüller, 1996; Martin \& Chao, 2001). A crucial test of whether activation of supplementary language areas is redundant or critical can be performed by temporarily influencing the activity state of neurons in supplementary language areas and recording its effect on the processing of specific word categories.

A unique possibility of studying the role of supplementary language areas in language processing is offered by the motor and premotor cortex. The premotor cortex is known to become active not only during motor tasks but also during action observation and listening to

Correspondence: Dr Friedemann Pulvermüller, as above.

E-mail: friedemann.pulvermuller@mrc-cbu.cam.ac.uk

Received 21 April 2004, revised 24 September 2004, accepted 29 October 2004 common action-related sounds (Gallese et al., 1996; Kohler et al., 2002). Spoken and written words can also activate the motor system and this activation can even be specific to semantic word types (Pulvermüller et al., 2001; Hauk \& Pulvermüller, 2004; Hauk et al., 2004; Shtyrov et al., 2004). Words related to actions involving different body parts, such as pick and kick, activate motor and premotor cortex in a somatotopic fashion so that, for example, the reading of leg-related words 'makes the motor humunculus move its feet' (de Lafuente \& Romo, 2004). This demonstrates categoryspecific links between the core language areas and motor representations in the processing of action words. The critical question of whether the information exchange is reciprocal and the activation in the motor system therefore also contributes to word processing is addressed here using transcranial magnetic stimulation (TMS), a technique causing reversible focal activity changes in the healthy human brain. We show for the first time that stimulation of the motor system influences language processing in a category-specific manner, thereby proving an active role of cortical motor systems in word recognition.

Hand and leg areas in the left hemisphere were stimulated using magnetic pulses below motor threshold while healthy right-handed native speakers of English read common arm- and leg-related words intermixed with meaningless pseudowords briefly flashed on a computer screen. Subjects had to respond by a brisk lip movement only when recognizing a meaningful word. Lip movements were chosen for lexical decision responses to minimize interference between semantic and motor preparation processes. Control experiments were performed with TMS delivered to the right non-dominant hemisphere and with sham stimulation. Subthreshold TMS of motor areas of the left hemisphere was predicted to specifically facilitate 
action word processing so that differential effects of arm and leg area stimulation would arise for arm- and leg-related words.

\section{Materials and methods Subjects}

Twelve subjects (mean age $\pm \mathrm{SD}, 26 \pm 4.8$ years; five females and seven males) participated in the study. Data from one subject had to be discarded due to strong electromyographic (EMG) baseline activity. All were native speakers of English and all except for one were monolinguals. All subjects were right handed (mean laterality quotient, $84 \pm 25$; Oldfield, 1971), had normal or corrected-to-normal vision and reported no history of neurological illness or drug abuse. Informed consent was obtained from all subjects and they were paid for their participation. The study was approved by the ethics committee of the Helsinki University Central Hospital.

\section{Stimuli}

The stimuli were 250 letter strings, including 50 arm- and 50 legrelated English words (e.g. fold, beat, grasp vs. kick, hike, step), 50 English distractor words and 100 pseudowords that were in accordance with the phonotactic and orthographic rules of English. The action word groups were matched for word length (average lengths were 4.5 letters for arm words and 4.6 for leg words) and standardized lexical and lemma frequency according to the CELEX database (average word frequency for arm words was 259 and for leg words 263 occurrences per million). A rating study (see Hauk \& Pulvermüller, 2004 for details) confirmed that the arm-related words elicited stronger associations of arm than leg actions in native speakers of English, whereas the opposite pattern was seen for leg words $\left(F_{1,49}=839.00, P<0.001\right)$. Furthermore, ratings of familiarity and imageability of the action words did not reveal differences between the stimulus groups. All of the action words included in the study could be used as verbs. As most words of English are lexically ambiguous (they may be used, for example, as nouns or verbs), this was also true for $86 \%$ of the words in the stimulus set (arm, 42 items; leg, 44 items).

\section{Stimulation procedure}

During the entire experiment, a fixation cross appeared in the middle of the screen, which was occasionally replaced by linguistic stimuli. Word and pseudoword stimuli were tachistoscopically presented, each for $100 \mathrm{~ms}$, in pseudorandom order with a stimulus onset asynchrony of $3 \mathrm{~s}$. TMS pulses were applied $150 \mathrm{~ms}$ after the onset of each linguistic stimulus. Letter strings were presented in white capital letters on a gray background in the middle of a computer screen at a distance of $2 \mathrm{~m}$ from the subjects' eyes. Letter strings subtended a horizontal visual angle smaller than $4^{\circ}$. Subjects were instructed to fixate their eyes on the fixation cross and to respond as quickly and as accurately as possible by a brisk lip movement to words only.

\section{Transcranial magnetic stimulation}

Hand and leg areas in the left language-dominant hemisphere were stimulated using single magnetic subthreshold pulses delivered by a figure-of-eight coil (Bailey et al., 2001). For arm and leg TMS, two successive runs were performed, each with half of the items from each stimulus category. Block order was counterbalanced over subjects. Stimulation was below motor threshold, at $90 \%$ of a value calculated as the weighted average of ipsi- and contralateral motor thresholds. The site where TMS elicited maximal electromyographic (EMG) responses of the contralateral dorsal interosseus muscle was used as the armrelated TMS site and the leg site was localized on the line connecting the arm site with the vertex, two thirds of its length away from the arm site. In a separate evaluation study, we found the leg locus determined in this manner to be $<1 \mathrm{~cm}$ away from the point where maximal EMG responses of the anterior tibialis muscle were elicited. The motor threshold was determined as the smallest intensity eliciting electromyograms with amplitudes of at least $50 \mu \mathrm{V}$ in five of 10 successive trials.

\section{Control conditions}

To investigate possible TMS-independent processing differences between the word groups under study, two control experiments were performed, one with right hemispheric stimulation and one with sham stimulation. The procedure for right hemispheric stimulation was the same as that detailed above. The order of right and left hemispheric stimulation was counterbalanced over subjects. The sham condition was always administered at the end. For sham stimulation, a 2-cmthick plastic block was placed between the TMS coil and scalp so that no relevant TMS of cortical tissue could occur. The sham condition served to mimic the auditory and somatosensory sensations produced by the mechanical vibration of the coil (Nikouline et al., 1999). Separate sham runs were performed for the left and right hemisphere, respectively. Subjects were not informed whether, in any given run, TMS or sham stimulation was applied.

\section{Recording of behavioral responses}

Subjects were instructed to respond by brisk mouth movements measured using two EMG electrodes fixed below and above the lips. The ground electrode was placed on the right cheek. The EMG signal was recorded using an amplifier whose input was blocked for $10 \mathrm{~ms}$ during and after the pulse by a sample-and-hold technique, thus avoiding the recording channels being saturated by the relatively high voltages induced by the TMS pulse (Virtanen et al., 1999). The sampling rate was $1450 \mathrm{~Hz}$ and the analog pass band filter was set to $0.1-500 \mathrm{~Hz}$.

\section{Data analysis}

For each participant, the average EMG amplitude was calculated and the first point in time exceeding $50 \%$ of the maximum value was defined as the onset of an overt response. The accuracy and average latency of these responses were calculated for each word group and subject. Reaction time values were z-transformed to remove intersubject variance. After chi-square tests performed to detect possible deviance from normal distribution, two-way Analyses of Variance (ANOVAs) with the factors Word group and Stimulation site were performed and $t$-tests were used for planned comparisons. Critical differences were also assessed using a non-parametric test, the Wilcoxon matched pairs test.

\section{Results}

Response latencies demonstrated a word-category-specific effect of TMS of left hemispheric arm and leg loci on the processing of arm and leg words $\left(F_{1,10}=16.2, P<0.002\right)$. This significant interaction of the factors Stimulation site and Word category was due to faster arm than 
leg word responses after arm site TMS $\left(t_{10}=2.2, P<0.03\right)$ and the reverse effect after TMS at the leg site $\left(t_{10}=2.7, P<0.01\right)$. Non-parametric testing using the Wilcoxon matched pairs test confirmed these results $(Z>2.1, P<0.03)$. This demonstrates a significant and specific effect of TMS of frontocentral motor systems in the left dominant hemisphere on the processing of arm- and legrelated words. Arm locus stimulation tended to lead to slower lexical decision responses (performed with the mouth) than leg area stimulation $\left(F_{1,10}=3.4, P=0.1\right)$, consistent with the closer proximity of face and arm motor representations in cortex as compared with the cortical face-leg distance. Control data obtained during right hemispheric stimulation and during sham stimulation did not reveal significant differences between word categories. A three-way ANOVA, for which data from left and right hemispheric TMS were pooled together (design: Hemisphere $\times$ Stimulation site $\times$ Word group), further confirmed the significant interaction of the factors Word group and Stimulation site $\left(F_{1,10}=14.1, P<0.005\right)$. The analysis of response accuracy revealed significantly more errors (misses) after left hemispheric compared with right hemispheric stimulation (11.2 vs. $\left.6.7 \% ; F_{1,10}=7.9, P<0.02\right)$. False-positive rates $(6.3 \%)$ did not differ between TMS conditions.

\section{Discussion}

We observed a differential effect of TMS of arm and leg areas in the left language-dominant hemisphere on the processing of arm and leg words. This supports category-specific functional links and information flow from motor areas to the cortical systems that process language. Action words do not only specifically activate arm- and legrelated motor and premotor areas (Pulvermüller et al., 2001; Hauk \& Pulvermüller, 2004; Hauk et al., 2004; Shtyrov et al., 2004); we also demonstrated that activity in the frontocentral cortex specifically contributes to the processing of action words of different semantic categories. This excludes the possibility that the coactivation of motor and premotor cortex is just a redundant by-product of action word processing and proves that sensorimotor areas can play a specific functional role in recognizing action words. This result is of general interest for cognitive brain theories because it provides strong support for category-specific distributed neuronal ensembles in the brain realizing cognitive operations (Martin \& Chao, 2001; Pulvermüller, 2001; Bogen \& Berker, 2002; Fuster, 2003).

When TMS was applied to the left hemispheric leg area, lexical decision responses to leg words were $35 \mathrm{~ms}$ faster than those for arm words. The difference in the opposite direction to arm area TMS was $14 \mathrm{~ms}$. This pattern can be explained by speeding of action word processing when the concordant motor site is stimulated. This is not the first study revealing speeding of language or other cognitive processes as a consequence of TMS (e.g. Mottaghy et al., 1999; Grosbras \& Paus, 2003). We suggest that, in the present study, the low stimulation intensity, about $90 \%$ of the motor threshold, was important for obtaining facilitation and that the asynchrony between the single TMS pulse and word onset $(150 \mathrm{~ms})$ was also relevant. In earlier studies

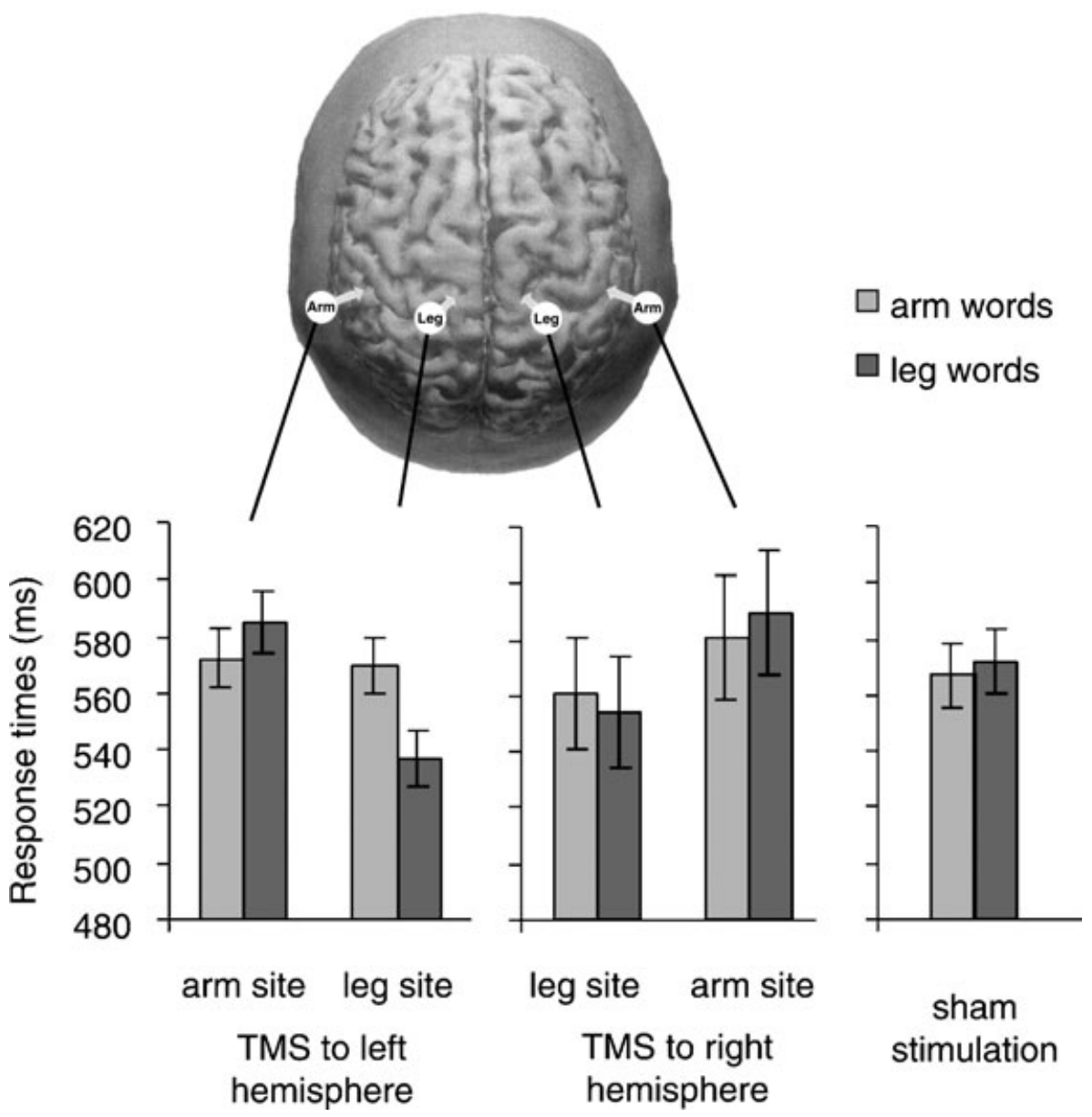

FIG. 1. Top, magnetic stimulation sites. Below left shows response times of lexical decisions on arm- and leg-related words made while TMS was applied to arm and leg loci in the left language-dominant hemisphere. Data from control experiments with TMS of the right hemisphere, and during sham stimulation are shown in the middle and right, respectively. 
using electroencephalography (EEG) and magnetoencephalography (MEG), neurophysiological responses differentiating between lexical items and pseudowords, as well as between lexical and semantic word categories, have been reported to be present within $200 \mathrm{~ms}$ of the onset of visual word presentation (for review see Pulvermüller, 2001; Sereno \& Rayner, 2003) and likewise the neurophysiological differences between action word subcategories were also present in this early time range (Hauk \& Pulvermüller, 2004; Shtyrov et al., 2004). Our choice of a stimulus-onset asynchrony (SOA) of $150 \mathrm{~ms}$ was motivated by these observations. Subthreshold TMS pulses applied shortly after word onset to areas that potentially play a role in the processing of word meaning may have a similar effect as priming by presentation of semantically related words (Kiefer, 2002).

Additional features of the results may inspire further interpretation. Arm word responses did not change between left hemispheric arm and leg site TMS, whereas the corresponding difference for leg words was pronounced. This could point to a stronger dependence of leg word processes on their semantic action-related features compared with that of arm words which could, in turn, be due to the greater variability or complexity of arm actions. Note that words such as 'fold', 'grasp' or 'write' refer to variable movements with very different features, whereas leg words such as 'run', 'walk' and 'step' refer to stereotypical actions that are more similar to each other. This may lead to stronger links between leg words and their action programs as compared with those of arm words. Although this is certainly a possibility, we feel that the absence of differences between stimulation sites for arm words should not be given too strong an interpretation because the left arm TMS site is close to the perisylvian language cortex, whereas the leg site is further away from it and this maydifferentially influence language processing in general or the articulator movements required in the task applied. Consistent with this, arm site TMS led to slower average responses than did leg site TMS. General slowing of responses for arm site TMS relative to leg site stimulation, which works against a between-site difference for arm words, could therefore underlie the similar latencies. We suggest that strong conclusions should not be drawn from the absence of a difference between arm and leg area TMS for arm word response latencies but rather from the significant interaction and the significant planned comparison tests between responses to matched stimulus groups when the same TMS locus was used.

Whereas left hemispheric TMS influenced lexical decision latencies significantly and specifically, no similar effects were apparent when TMS was applied to the right hemisphere, where average differences between conditions were generally smaller and standard errors (SEs) tended to be larger. All subjects were strongly right handed, as revealed by neuropsychological testing, and it is known that right-handedness is usually associated with left hemispheric dominance for language (see, e.g. Knecht et al., 2000). The effect of left hemispheric TMS is therefore best explained by interactions with cortical language networks lateralized to the left hemisphere. However, earlier work suggested specific contributions of right hemispheric circuits to word processing in general (Zaidel, 1976; Mohr et al., 1994) and word-category-specific processes in particular (Chiarello et al., 1990; Grossman et al., 2002; Neininger \& Pulvermüller, 2003; Hauk et al., 2004). Consistent with this, average reaction times after right hemispheric TMS showed a tendency in the same direction as the statistically documented effect after TMS to the left dominant hemisphere (Fig. 1) and pooling of data from TMS to both hemispheres replicated the significant interaction obtained for the dominant left hemisphere alone. Therefore, although no strong evidence for right hemispheric TMS effects on language processing was obtained,
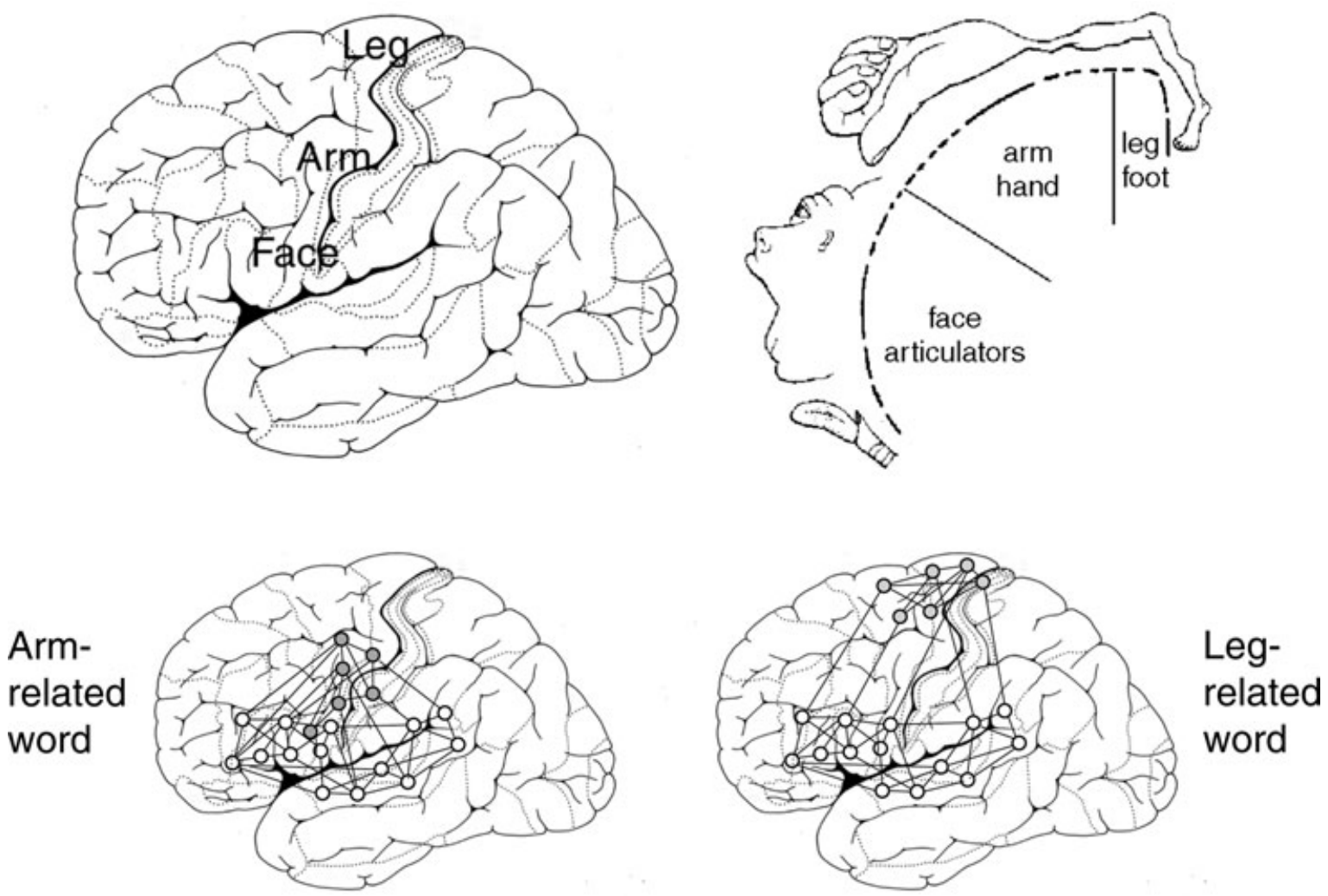

FIG. 2. Somatotopic organization of the motor cortex according to Penfield \& Rassumssen (1950). Model of distributed cell assemblies for action words semantically related to the arm (left) and leg (right). Neuron ensembles distributed over perisylvian core language areas and arm or leg motor and premotor cortex are assumed to represent and process arm and leg words, respectively. 
the present data do not argue against a possible contribution of right hemispheric circuits to language processing in right-handed subjects. The fact that right hemispheric TMS did not significantly influence lexical decision latencies in the present study may be due to the additional synaptic step for callosal crossing required for information exchange between the right hemispheric arm/leg areas and the left hemispheric perisylvian core language areas.

The differential TMS influence on action words is best explained in terms of neuronal assemblies binding language and action at the cortical level. These networks may be distributed over core language areas, in inferior frontal and superior temporal cortex where word forms are stored, and additional supplementary language areas in the motor system storing action-related aspects of word meaning (Fig. 2; Pulvermüller et al., 2001), which may include mirror neurons with a role in both action and perception (Fadiga et al., 2002; Rizzolatti et al., 2002). This model suggests that, for words related to arm and leg actions, the categoryspecific semantic neurons are located in different parts of the motor system, lateral for arm and dorsal for leg actions. Against this background, we interpret our results as evidence for specific functional links from supplementary language cortex in motor or premotor cortex to core language areas in the processing of words related to actions.

The picture of cortical function arising from these data is that of the cortex as a storehouse of words and their meanings bound together by distributed neuronal systems with specific topographies. These neuronal ensembles, which may have formed during ontogenesis as a consequence of genetic factors and experience, would be spread out over classic core language areas, where all word-related assemblies have some of their neurons, and specific semantically related neurons in supplementary language areas, e.g. in sensorimotor cortex. This view opens an avenue for reconciling classic modular language models with distributed models and can account for both challenges of the cognitive neuroscience of language, the predominance of aphasias after left perisylvian lesions and the widespread activation in a range of language tasks revealed by neuroimaging (Pulvermüller, 2003).

\section{Acknowledgements}

We thank Chris Bailey, Giovanni Buccino, Luigi Cattaneo, Massimo Gangitano, Dubravko Kicic, Tuomas Murdoch, Giacomo Rizzolatti and Yury Shtyrov for help at different stages of this work. The insightful comments and helpful suggestions by two anonymous referees are also thankfully acknowledged. The study was supported by the Medical Research Council (UK), University of Helsinki and European Community under the 'Information Society Technologies Programme' (IST-2001-35282).

\section{Abbreviations}

ANOVA, analysis of variance; EEG, electrocencephalography/graphic; EMG, electromyography/graphic; MEG, magnetoencephalography/graphic; SE, standard error; SOA, stimulus onset asynchrony; TMS, transcranial magnetic stimulation.

\section{References}

Bailey, C.J., Karhu, J. \& Ilmoniemi, R.J. (2001) Transcranial magnetic stimulation as a tool for cognitive studies. Scand. J. Psychol., 42, 297-305.

Binder, J.R., Frost, J.A., Hammeke, T.A., Cox, R.W., Rao, S.M. \& Prieto, T. (1997) Human brain language areas identified by functional magnetic resonance imaging. J. Neurosci., 17, 353-362.

Bogen, J.E. \& Berker, E. (2002) Face modules, face network: the cognitive architecture of the brain revealed through studies of face processing. Neurology, 59, 652-653; author reply 653.

Chiarello, C., Burgess, C., Richards, L. \& Pollock, A. (1990) Semantic and associative priming in the cerebral hemispheres: some words do, some words don't...sometimes, some places. Brain Lang., 38, 75-104.
Fadiga, L., Craighero, L., Buccino, G. \& Rizzolatti, G. (2002) Speech listening specifically modulates the excitability of tongue muscles: a TMS study. Eur. J. Neurosci., 15, 399-402.

Fuster, J.M. (2003) Cortex and Mind: Unifying Cognition. Oxford University Press, Oxford.

Gallese, V., Fadiga, L., Fogassi, L. \& Rizzolatti, G. (1996) Action recognition in the premotor cortex. Brain, 119, 593-609.

Geschwind, N. (1970) The organization of language and the brain. Science, 170, 940-944.

Grosbras, M.H. \& Paus, T. (2003) Transcranial magnetic stimulation of the human frontal eye field facilitates visual awareness. Eur. J. Neurosci., 18, $3121-3126$.

Grossman, M., Koenig, P., DeVita, C., Glosser, G., Alsop, D., Detre, J. \& Gee, J. (2002) The neural basis for category-specific knowledge: an fMRI study. Neuroimage, 15, 936-948.

Hauk, O. \& Pulvermüller, F. (2004) Neurophysiological distinction of action words in the fronto-central cortex. Hum. Brain Mapp., 21, 191-201.

Hauk, O., Johnsrude, I. \& Pulvermüller, F. (2004) Somatotopic representation of action words in the motor and premotor cortex. Neuron, 41, 301-307.

Kiefer, M. (2002) The N400 is modulated by unconsciously perceived masked words: further evidence for an automatic spreading activation account of N400 priming effects. Cogn. Brain Res., 13, 27-39.

Knecht, S., Drager, B., Deppe, M., Bobe, L., Lohmann, H., Floel, A., Ringelstein, E.B. \& Henningsen, H. (2000) Handedness and hemispheric language dominance in healthy humans. Brain, 123 (12), 2512-2518.

Kohler, E., Keysers, C., Umilta, M.A., Fogassi, L., Gallese, V. \& Rizzolatti, G. (2002) Hearing sounds, understanding actions: action representation in mirror neurons. Science, 297, 846-848.

de Lafuente, V. \& Romo, R. (2004) Language abilities of motor cortex. Neuron, 41, 178-180.

Lichtheim, L. (1885) On aphasia. Brain, 7, 433-484.

Martin, A. \& Chao, L.L. (2001) Semantic memory and the brain: structure and processes. Curr. Opin. Neurobiol., 11, 194-201.

Mohr, B., Pulvermüller, F. \& Zaidel, E. (1994) Lexical decision after left, right and bilateral presentation of content words, function words and nonwords: evidence for interhemispheric interaction. Neuropsychologia, 32, 105-124.

Mottaghy, F.M., Hungs, M., Brugmann, M., Sparing, R., Boroojerdi, B., Foltys, H., Huber, W. \& Topper, R. (1999) Facilitation of picture naming after repetitive transcranial magnetic stimulation. Neurology, 53, 1806-1812.

Neininger, B. \& Pulvermüller, F. (2003) Word-category specific deficits after lesions in the right hemisphere. Neuropsychologia, 41, 53-70.

Nikouline, V., Ruohonen, J. \& Ilmoniemi, R.J. (1999) The role of the coil click in TMS assessed with simultaneous EEG. Clin. Neurophysiol., 110, 13251328.

Oldfield, R.C. (1971) The assessment and analysis of handedness: the Edinburgh Inventory. Neuropsychologia, 9, 97-113.

Penfield, W. and Rasmussen, T. (1950) The Cerebral Cortex of Man. Macmillan, New York.

Pulvermüller, F. (1996) Hebb's concept of cell assemblies and the psychophysiology of word processing. Psychophysiology, 33, 317-333.

Pulvermüller, F. (2001) Brain reflections of words and their meaning. Trends Cogn. Sci., 5, 517-524.

Pulvermüller, F. (2003) The Neuroscience of Language. Cambridge University Press, Cambridge.

Pulvermüller, F., Hummel, F. \& Härle, M. (2001) Walking or talking? Behavioral and neurophysiological correlates of action verb processing. Brain Lang., 78, 143-168.

Rizzolatti, G., Fogassi, L. \& Gallese, V. (2002) Motor and cognitive functions of the ventral premotor cortex. Curr. Opin. Neurobiol., 12, 149-154.

Sereno, S.C. \& Rayner, K. (2003) Measuring word recognition in reading: eye movements and event-related potentials. Trends Cogn. Sci., 7, 489-493.

Shallice, T. (1988) From Neuropsychology to Mental Structure. Cambridge University Press, New York.

Shtyrov, Y., Hauk, O. \& Pulvermüller, F. (2004) Distributed neuronal networks for encoding category-specific semantic information: the mismatch negativity to action words. Eur. J. Neurosci., 19, 1083-1092.

Vandenberghe, R., Price, C., Wise, R., Josephs, O. \& Frackowiak, R.S. (1996) Functional anatomy of a common semantic system for words and pictures. Nature, 383, 254-256.

Virtanen, J., Ruohonen, J., Naatanen, R. \& Ilmoniemi, R.J. (1999) Instrumentation for the measurement of electric brain responses to transcranial magnetic stimulation. Med. Biol. Eng. Comput., 37, 322-326.

Zaidel, E. (1976) Auditory vocabulary of the right hemisphere following brain bisection or hemidecortication. Cortex, 12, 191-211. 